\title{
Éditorial
}

\section{Stratégie pour l'avenir de l'épuration des eaux de l'agglomération parisienne}

Les bases du réseau actuel des égouts de Paris datent de 1856, lorsque le baron Haussmann, préfet de la Seine, approuva la réalisation des travaux proposés par BELGRAND. L'originalité de ce programme consistait à prévoir le rejet des eaux usées en dehors des limites de l'agglomération au lieu de les évacuer en Seine, au plus près. BELGRAND préconisa de diriger les effluents en aval de Paris à la hauteur de Clichy par quatre grands collecteurs qui formèrent les artères maîtresses du réseau d'égouts parisien. Il en résulta une pollution notable de la Seine à l'aval de Clichy et rapidement il devint nécessaire d'envisager l'épuration des eaux d'égout avant leur rejet en fleuve. Une nouvelle étape de l'assainissement parisien s'est donc développée. A la suite d'expériences concluantes menées dans la plaine de Gennevilliers, ils envisagèrent de traiter les effluents par la voie de l'épandage agricole sur des terrains filtrants convenablement drainés. Ceci aboutit entre 1895 et 1905 à la mise en service progressive des champs d'épandage d'Achères, acquis par la Ville de Paris et alimentés depuis Clichy par l'émissaire général. Il fut alors possible, grâce à leur efficacité de faire bénéficier les parisiens d'un véritable «tout à l'égout» dont le raccordement fut d'ailleurs rendu obligatoire par la loi du 10 juillet 1894. En 1919, la surface des champs d'épandage en activité atteignait 5000 ha. Elle permettait à l'époque de traiter l'ensemble des eaux usées recueillies. Cette situation fut satisfaisante jusqu'aux lendemains de la première guerre mondiale.

Par suite de l'évolution démographique de la région parisienne, surtout de l'urbanisation et de l'industrialisation de la banlieue, de l'accroissement des volumes d'eaux consommés, des champs d'épan- 
dage de la Ville de Paris devinrent insuffisants pour assurer l'assainissement de l'agglomération parisienne. Les problèmes de l'assainissement reprirent de l'acuité et durent être repensés. Ils se transposèrent d'ailleurs du cadre strictement parisien à un cadre régional lié à la topographie de la région parisienne et intéressant les départements de la Seine et de la Seine-et-Oise. Ils conduisirent en 1930 à l'élaboration du programme général d'assainissement, véritable charte de l'assainissement de la région parisienne.

Ce programme, dont la réalisation s'est poursuivie d'une manière continue, tendait à rassembler la quasi-totalité des eaux d'égout de cette agglomération sur une station d'épuration unique, implantée en tête des champs d'épandage de la Ville de Paris dans la plaine d'Achères (entre la forêt de Saint-Germain et la Seine).

Par ailleurs, deux autres stations drainent les eaux usées des départements situés à l'amont de Paris: les stations de Valenton dans l'Essonne et de Noisy-le-Grand en Seine-Saint-Denis. L'ensemble de ces travaux est géré par un organisme interdépartemental: le Syndicat Interdépartemental d'Assainissement de l'Agglomération Parisienne établi sur 7 départements et desservant plus de 10 millions d'habitants.

Longtemps le grand public n'a vu dans l'épuration des eaux que le moyen de préserver la faune et la flore des rivières, mais il commence désormais à être sensible au fait qu'épurer les eaux usées c'est aussi et avant tout préserver une ressource naturelle indispensable à la vie humaine.

Lorsque nous énonçons "Rendre l'eau à la vie, c'est notre devoirfaire". Nous pensons en effet, qu'à Orly, Choisy et Ivry, en aval de la station d'épuration de Valenton, sont implantées trois des plus importantes usines de production d'eau potable de l'agglomération parisienne. En outre, les prises d'eau de l'usine d'Aubergenville, qui alimentent une grande partie du département des Yvelines, se situent quant à elles en aval de celle-ci.

Sachant que la consommation d'eau dans l'agglomération parisienne représente un volume proche de la moitié du débit moyen de la Seine à l'étiage $\left(70 \mathrm{~m}^{3} / \mathrm{s}\right)$ il est évident que si nous ne faisions pas correctement notre travail d'assainissement, les rejets viendraient souiller gravement l'eau de rivière dont la mauvaise qualité ne permettrait plus alors son retraitement pour produire de l'eau potable. Si l'on compare le débit moyen de la Seine à Paris $\left(300 \mathrm{~m}^{3} / \mathrm{s}\right)$ à celui du Saint-Laurent au Canada qui est de $10200 \mathrm{~m}^{3} / \mathrm{s}$ ou à celui des autres fleuves d'Amérique comme l'Amazone (200 $\left.000 \quad \mathrm{~m}^{3} / \mathrm{s}\right)$, d'Afrique ou encore d'Asie comme l'Angara (2 $900 \mathrm{~m} / \mathrm{s}$ ) notre " fleuve" apparaît bien modeste et fragile. Nous sommes donc obligés de gérer ce faible potentiel sur le fil du rasoir car tout incident sérieux 
de fonctionnement au niveau d'une seule station d'épuration poserait immanquablement de graves problèmes pour ce qui concerne la qualité de l'approvisionnement en eau de la Région Ile-de-France.

Il est donc impérativement de notre devoir d'utiliser tout notre savoir-faire pour régénérer les eaux usées avant de les rendre au fleuve.

Il convient également de prendre en compte à ce stade le rôle important des barrages-réservoirs du bassin de la Seine qui permettent de réguler le débit de l'eau et de la stocker pour pouvoir continuer à approvisionner les consommateurs dans de bonnes conditions, même en cas de sécheresse, ce qui a été le cas en 1991, 1992 et 1993.

Producteurs d'eau, assainisseurs et gestionnaires des barragesréservoirs forment une grande famille solidaire qui permet d'assurer à l'ensemble des habitants de la région parisienne une alimentation en eau de très grande qualité qui a la réputation d'être tout à fait exceptionnelle.

Pour maintenir celle-ci, il a été créé un programme "Seine Propre» qui comportait plusieurs volets:

- La modernisation des deux premières tranches et du prétraitement de la station d'épuration d'Achères dont la capacité sera maintenue à $2100000 \mathrm{~m}^{3 / j}$.

- La construction de l'émissaire Sèvres-Achères, branche de Nanterre, destiné à collecter les rejets encore existants en Seine sur son parcours en interceptant notamment les eaux du ru de Marivel qui se déversaient jusqu'alors au niveau du Pont de Sèvres.

- La construction à Valenton en amont de Paris d'une nouvelle station d'épuration d'une capacité de $300000 \mathrm{~m} 3 / \mathrm{j}$.

Ces trois premières opérations ont été menées à bien. Des capacités de traitement complémentaires ont été recherchées sur d'autres sites dans le cadre du schéma directeur d'assainissement «Horizon 2015 », notamment à Colombes, où vient de débuter la réalisation d'une station de haute technologie capable de traiter $240000 \mathrm{~m}^{3}$ par jour par temps sec.

Dans le cadre de ce programme, nous avons entrepris également des travaux pour réduire les nuisances olfactives et sonores, notamment à Achères.

En ce qui concerne la répartition des ouvrages on constate lorsqu'on regarde une carte du réseau SIAAP, qu'il existe un déséquilibre entre l'Est et l'Ouest de l'agglomération, ceci pour des raisons historiques. Un des buts du nouveau schéma directeur est donc de rééqui- 
librer les moyens d'épuration tout en les complétant pour assurer le traitement de la totalité des eaux usées. Cette nouvelle politique qui avait été amorcée avec la construction de la station d'épuration de Valenton sera poursuivie avec le doublement de cette station (600 $000 \mathrm{~m}^{3} / \mathrm{j}$ ) et l'extension de celle de Noisy-le-Grand qui sera portée de 20000 à $60000 \mathrm{~m}^{3} / \mathrm{j}$. Nous envisageons aussi la construction d'une nouvelle unité d'épuration des eaux en Seine-Saint-Denis. Ces différentes opérations permettront de disposer d'une capacité journalière de traitement totale de $3325000 \mathrm{~m}^{3}$ par jour en incluant une marge de sécurité de $10 \%$ et qui correspond aux besoins d'épuration des eaux usées par temps sec à l'horizon 2015.

Mais la protection du milieu naturel exige que nous allions plus loin encore, en traitant les eaux de pluie dont les premiers flots ont lessivé les chaussées et les toits et représentent un risque élevé de pollution. Nous allons aménager des déversoirs d'orages, et doter les stations d'épuration de moyens destinés à faire subir aux eaux excédentaires de temps de pluie un prétraitement efficace pour minimiser leur impact sur le milieu naturel. Nous utiliserons des procédés techniques différents. A Colombes, ce seront des cultures fixées, à Achères, la clarifloculation afin de nous adapter aux structures déjà existantes.

Toutefois, les débits instantanés par temps de pluie étant considérables, nous devrons construire des bassins de stockage de ces eaux afin de pouvoir les traiter progressivement. Nous avons à cet effet un projet important en Seine-Saint-Denis, sur le site du grand stade, qui se situe à la convergence d'un certain nombre de réseaux du SIAAP où nous allons construire un bassin de stockage. Nous en sommes actuellement à la phase de pré-étude.

Comme on peut le constater le nouveau schéma directeur d'assainissement a fixé l'ensemble des objectifs à atteindre d'ici 2015. Son coût est actuellement estimé à 25 milliards de francs, dont 9 milliards qui devront être engagés d'ici 1997: 7 pour les stations d'épuration et 2 pour les émissaires. Cependant, sans modifier la stratégie globale, les incertitudes sur l'évolution des quantités d'eau qui devront être traitées dans les années à venir pourront conduire à des actualisations de ce schéma.

Dans l'état actuel des choses, il est prévu site par site:

- Colombes : création d'une nouvelle usine capable de traiter par temps sec $240000 \mathrm{~m}^{3}$ par jour à un niveau correspondant aux normes européennes les plus sévères, et par temps de pluie un débit quatre fois plus important avec des performances demeurant remarquables. L'intégration de cette unité dans le site en bordure de Seine a été particulièrement soignée, de même que la protection de son 
environnement puisqu'elle sera entièrement close et l'air désodorisé avant d'être rejeté à l'atmosphère.

- Achères: dans un premier temps, poursuite des travaux antinuisances dans le cadre de la construction d'une unité de traitement des eaux pluviales par clarifloculation. La clarifloculation devrait aussi permettre de traiter le phosphore présent dans les eaux tant par temps sec que par temps de pluie.

Par ailleurs, trois prototypes de $30000 \mathrm{~m}^{3}$ sont expérimentés pour le traitement de la pollution azotée et le procédé sera étendu progressivement à l'ensemble de la station.

- Valenton: doublement de sa capacité et travaux d'environnement (couverture, insonorisation, désodorisation). A côté de ces projets d'ores et déjà engagés, citons les projets à plus long terme:

- Noisy-le-Grand: extension de la capacité de la station.

- La Morée, en Seine-Saint-Denis: création d'une nouvelle station.

- Les Grésillons : à l'extrémité de l'aqueduc d'Achères, une station d'une capacité maximale de $300000 \mathrm{~m}^{3}$ par jour sera réalisée.

En marge de ces opérations «lourdes», le SIAAP s'est attaché à ce que nous appelons la pollution visuelle, à savoir les déchets flottants sur la Seine.

Quatre barrages destinés à capturer ces flottants sont déjà opérationnels et six autres le seront prochainement. Nous disposons désormais d'un bateau qui aura pour mission la récupération des déchets (bois, plastique...) retenus par ces barrages flottants arrimés près des ponts.

Nous avons aussi décidé de mettre en place un certain nombre de dispositifs d'oxygénation, destinés à réduire en période de crise (gros orages) la mortalité des poissons évoluant dans la Seine. La technique consiste à dissoudre de l'oxygène pur sous pression dans l'eau, de façon à créer des «îlots de survie» pour les poissons pendant ces périodes.

L'ensemble de ces données traduit l'effort constant et considérable que les assainisseurs sont contraints à mettre en œuvre aujourd'hui comme par le passé pour restaurer la qualité des eaux et tenter de donner en héritage aux population futures l'équivalent de celui reçu de leurs illustres prédécesseurs. 